\title{
Synapse Loss in Olfactory Local Interneurons Modifies Perception
}

\author{
Angel Acebes, Alfonso Martín-Peña, Valérie Chevalier, and Alberto Ferrús \\ Cajal Institute, Consejo Superior de Investigaciones Científicas, 28002 Madrid, Spain
}

Synapse loss correlates with cognitive decline in aging and most neurological pathologies. Sensory perception changes often represent subtle dysfunctions that precede the onset of a neurodegenerative disease. However, a cause-effect relationship between synapse loss and sensory perception deficits is difficult to prove and quantify due to functional and structural adaptation of neural systems. Here we modified a PI3K/AKT/GSK3 signaling pathway to reduce the number of synapses - without affecting the number of cells - in five subsets of local interneurons of the Drosophila olfactory glomeruli and measured the behavioral effects on olfactory perception. The neuron subsets were chosen under the criteria of GABA or ChAT expression. The reduction of one subset of synapses, mostly inhibitory, converted the responses to all odorants and concentrations tested as repulsive, while the reduction of another subset, mostly excitatory, led to a shift toward attraction. However, the simultaneous reduction of both synapse subsets restored normal perception. One group of local interneurons proved unaffected by the induced synapse loss in the perception of some odorants, indicating a functional specialization of these cells. Using genetic tools for space and temporal control of synapse number decrease, we show that the perception effects are specific to the local interneurons, rather than the mushroom bodies, and are not based on major structural changes elicited during development. These findings demonstrate that synapse loss cause sensory perception changes and suggest that normal perception is based on a balance between excitation and inhibition.

\section{Introduction}

Stimuli captured by sensory neurons need to be orderly processed from first-order neuropiles onwards. For odorants, this neuropile is the olfactory bulb of vertebrates or its analogous antennal lobe (AL) of invertebrates, where sensory inputs elicit specific patterns of glomerular activation (Rubin and Katz, 1999; Uchida et al., 2000; Wachowiak and Cohen, 2001; Ng et al., 2002; Wang et al., 2003; Wilson et al., 2004; Lin et al., 2006; Masse et al., 2009; Su et al., 2009). At least two types of local interneurons (LNs), expressing GABA or acetyl choline, interconnect glomeruli within the AL (Stocker et al., 1997; Wilson and Laurent, 2005; Shang et al., 2007). LNs show specific firing profiles under patch-clamp recordings (Seki et al., 2010). Excitatory local interneurons (eLNs) receive monosynaptic input from sensory cells and establish reciprocal connections with other eLNs (Huang et al., 2010). Lateral inhibition between glomeruli, mediated by inhibitory local interneurons (iLNs), has been interpreted as a mechanism to enhance contrast and facilitate discrimination between stimuli (Yokoi et al., 1995; Arevian et al., 2008). Likewise, lateral excita-

\footnotetext{
Received Sept. 27, 2010; revised Dec. 13, 2010; accepted Dec. 17, 2010.

This research was funded by grants from the Ministry of Research (BFU2009-12410/BMC), Fundación CIEN, and a Network of Excellence from the Comunidad de Madrid (OLFACTOSENSE) (SEM-0255-2006-02). We are grateful for the cooperation of Ronald Davis (Baylor College of Medicine, Houston, TX), in whose laboratory the functional imaging experiments were carried out. We are in debt to Liqun Luo (Stanford University, Stanford, CA), who kindly provided his collection of olfactory Gal4 lines. Also, we thank the Bloomington Stock Center for fly strains.

Correspondence should be addressed to Alberto Ferrús, Cajal Institute, Consejo Superior de Investigaciones Científicas, Avenida Dr. Arce 37, 28002 Madrid, Spain. E-mail: aferrus@cajal.csic.es.

A. Martín-Peña's present address: Department of Neuroscience, The Scripps Research Institute, 130 Scripps Way, Jupiter, FL 33458.

DOI:10.1523/JNEUROSCI.5046-10.2011

Copyright $\odot 2011$ the authors $\quad 0270-6474 / 11 / 312734-12 \$ 15.00 / 0$
}

tion has been proposed to boost signal transmission toward higher brain centers (Olsen et al., 2007; Shang et al., 2007; Olsen and Wilson, 2008). Interglomerular inhibition is thought to dominate over interglomerular excitation for gain control of olfactory stimuli (Olsen and Wilson, 2008). The coexistence of eLNs and iLNs in the same glomeruli, however, calls for a joint functional role in the elaboration of odorant perception. Indeed, recent evidence indicates that eLNs and iLNs are mutually connected through chemical (cholinergic and GABAergic, respectively) as well as electrical (Yaksi and Wilson, 2010) synapses. In agreement with these functional considerations, LNs are morphologically heterogeneous (Chou et al., 2010).

In the human cortex, pyramidal neuron activity is modulated by a balance between converging inhibitory and excitatory terminals, and deviations in either direction are hypothesized to cause psychosis or seizure (Gan et al., 2003; Ben-Ari, 2006; Daskalakis et al., 2007; Dudek and Sutula, 2007). Loss of inhibitory synapses has been associated with schizophrenia and bipolar disorder (Benes and Berretta, 2001; Ross et al., 2006), while loss of excitatory synapses appears associated with Alzheimer and aging (Geinisman et al., 2004; Spires and Hyman, 2004). Whether these synapse counts made on postmortem, clinically treated, brains are cause or effect of the pathology is still a matter of debate.

In this report, we use the olfactory glomeruli of Drosophila as a neural system in which to assay the sensory perception and behavioral effects of genetically induced synapse loss in specific neurons. Previous studies have made use of genetic tools (e.g., tetanus toxin or thermosensitive dynamin expression) to fully silence specific neurons in the olfactory and other brain centers (Kitamoto, 2001; Keller et al., 2002). This approach is useful to 

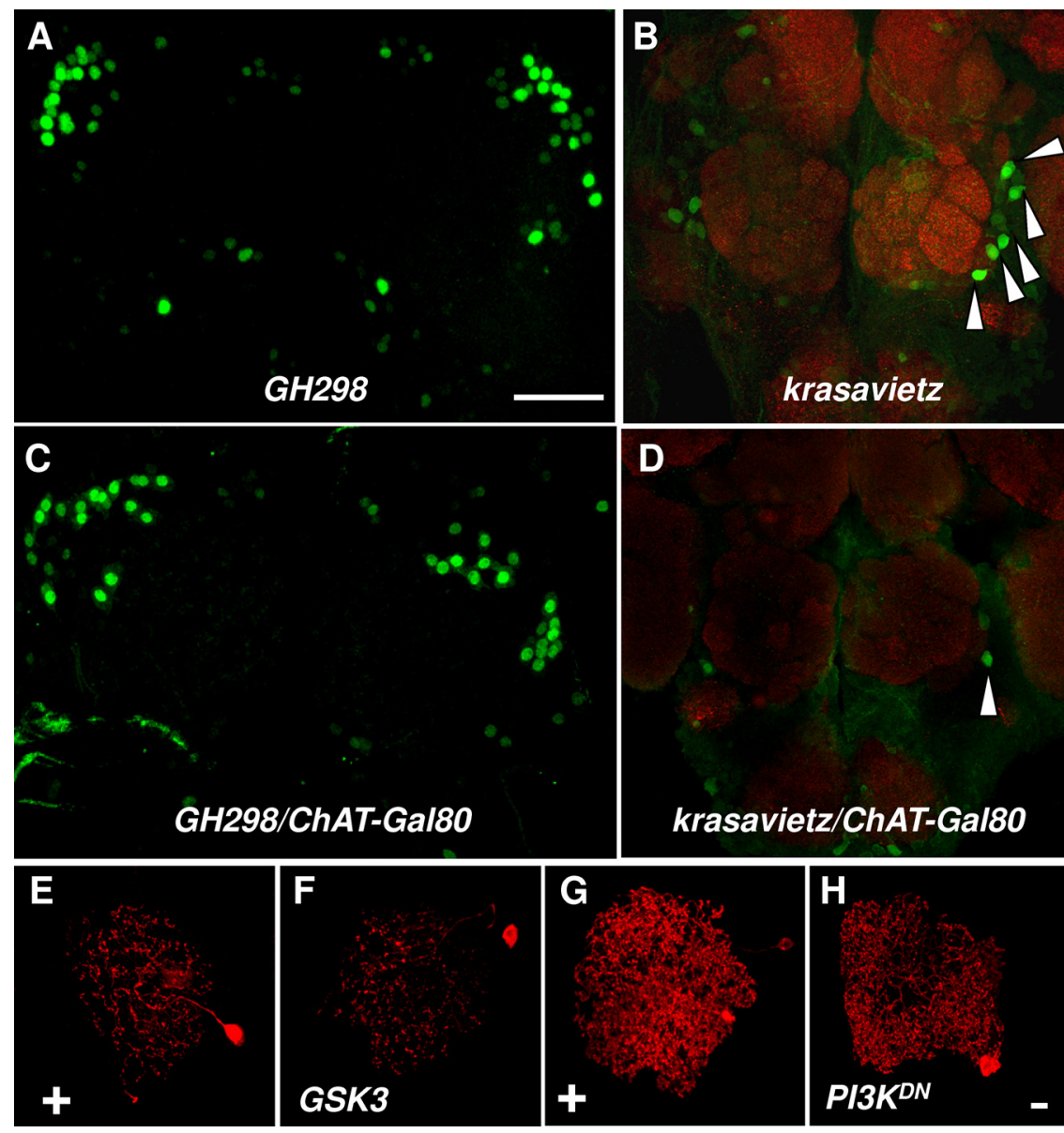

Figure 1. Local antennal lobe neurons and the morphological effects of upregulating GSK3 or PI3K ${ }^{\mathrm{DN}}$. $\boldsymbol{A}$, Expression domain of GH298-Gal4 visualized by the nuclear reporter GFP $P^{n / s}$. B, Expression domain of krasavietz-Gal4 visualized by GFP ${ }^{n / s}$ and counterstained with the monoclonal antibody nc82 to reveal the synapses (Wagh et al., 2006). C, Coexpression of the Gal 4 repressor, Gal80, under the $3.3 \mathrm{~kb}$ enhancer from the choline acetyl transferase gene (ChAT) (genotype: GH298-Gal4>UAS-GFPn/s; ChAT> Gal80). Note that very few GH298-Gal4 cells have been silenced, indicating that most neurons in this domain of expression are not excitatory (see quantification in Table 1). D. Coexpression of ChAT-Gal80 (genotype: krasavietz-Gal4 > UAS-GFPn/s; ChAT> Gal80) Note that most neurons have suppressed the Gal4 expression, indicating that most of them are cholinergic. Neuron somata are indicated by arrowheads on the left side of each brain $(\boldsymbol{B}, \boldsymbol{D})$. $\boldsymbol{E}-\boldsymbol{H}$, Single neurons from $h \mathrm{FFLP/Gal4}>$ UAS-FRT-CD2-FRT-CD8-GFP adults that had been heat shocked $\left(37^{\circ} \mathrm{C}\right)$ for $55 \mathrm{~min}$ at the late third-instar larval stage (Nellen et al., 1996). The GFP reporter was revealed by anti-GFP and Alexa 568 on whole-mount brains. $\boldsymbol{E}$, Normal control of a local inhibitory interneuron (GH298-Gal4). $\boldsymbol{F}$, Effect of driving UAS-GSK3. G, Normal control of a local excitatory interneuron (krasavietz-Gal4). $\boldsymbol{H}$, Effect of driving UAS-PI3K $K^{D N}$. Note the reduced branching in both types of neurons caused by, either the upregulation of GSK3 or the downregulation of $P I 3 K$. Scale bars: $\boldsymbol{A}-\boldsymbol{D}$ (in $\boldsymbol{A}), 50 \mu \mathrm{m} ; \boldsymbol{E}-\boldsymbol{H}$ (in $\boldsymbol{H}$ ), $10 \mu \mathrm{m}$. Laser settings in the confocal microscope were kept constant in all control versus experimental comparisons.

unveil the role of the affected neurons in the behavioral task under consideration. However, the strategy is unrealistic with respect to most neural pathologies, in particular for the initial symptoms, because synaptic function deteriorates progressively, rather than abruptly, and well before neurons are lost (Selkoe, 2002; Scheff and Price, 2006; Helton et al., 2008). Here we attenuate, rather than silence, the activity of the selected neurons aiming to gain a more faithful image of aging and early steps of pathological conditions. To reduce the number of synapses, we upregulated the levels of GSK3 or downregulated those of PI3K, two elements of a novel synaptogenic pathway that we identified in Drosophila and that seems to be conserved in vertebrates (Martín-Peña et al., 2006). The choice of GSK3 is also appropriate because a correlation has been established between high levels of this enzyme and the severity of cognition deficits in Alzheimer patients (Hooper et al., 2008).

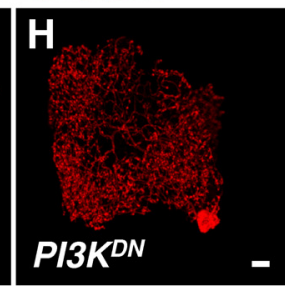

\section{Materials and Methods}

Fly strains. Fly strains GH298-Gal4 and $\mathrm{Hs}$ flp; Sp/CyO; UAS-frtCD2y frtCD8-GFP were from Dr. R. F. Stocker (University of Fribourg, Fribourg, Switzerland) (Wong et al., 2002); line krasavietz-Gal4 was from Dr. G Miessenbock (Oxford University, Oxford, UK) (Dubnau et al., 2003; Shang et al., 2007); another krasavietz-Gal4 was provided by Dr. L. Luo (Stanford University, Palo Alto, CA) (Chou et al., 2010), which is referred to here as Line9Gal4, since its domain of expression is clearly different from the previous one; lines LN1Gal4 (NP1227) and LN2-Gal4 (NP2426) were provided by Dr. S. Sachse (MPI, Jena, Germany) and first reported by K. Ito (Tokyo University, Tokyo, Japan) (Okada et al., 2009; Tanaka et al., 2009); line UAS-PI3K92E ${ }^{D 954 A}$, carrying a dominant-negative PI3K mutation and referred to in the text as $U A S-P I 3 K^{D N}$, was provided by Dr. S. Leevers (Cancer Research Center, London, UK) (Leevers et al., 1996); and UAS-Shaggy (Sgg/GSK3) was from Dr. M. Calleja (Center for Molecular Biology, CBM, Madrid, Spain) (Martín-Peña et al., 2006). The Drosophila gene Shaggy is homologous to Glycogen synthase kinase 3 (GSK3) in vertebrates (Franco et al., 2004). The UAS-CD8-GFP and ChAT ${ }^{3.3}$ Gal80 lines were from Dr. J. F. Ferveur (University of Burgundy, Dijon, France) (Kitamoto et al., 1992), UAS-CD2-HRP was from Dr. L. Luo (HHMI Stanford University) (Watts et al., 2004), and UAS-GFP ${ }^{\text {nls }}$ was from the stock center in Bloomington (Fly Base, http://flybase.bio.indiana.edu). Lines Galso ${ }^{t s}$, $M B G a l 80$, and UAS-G-CaMP were from Dr. R. Davis (Baylor College, Houston, TX) (McGuire et al., 2003; Wang et al., 2003; Krashes et al., 2007); 201Y-Gal4 line (expressed in most neurons of the $\gamma$ lobe and a small subset of $\alpha$ and $\beta$ lobe neurons) was from Dr. M. L. Parmentier (IGF, Montpellier, France) (Kraft et al., 1998); line MB247-Gal4 (corresponding to the Dmef2 gene and expressed in most neurons of the mushroom body) was from Dr. M. Heisenberg (Würzburg University, Würzburg, Germany) (Schwaerzel et al., 2002); and $\mathrm{TH}$ Gal80 line was from Dr. Scott Waddell (University of Massachusetts Medical School, Worcester, MA) (Sitaraman et al., 2008).

Electron microscopy. Transmission electron microscopy (TEM) was performed in 7-d-old CD2-HRP-expressing flies anesthetized on ice, fixed in $0.1 \mathrm{M}$ phosphate buffer $/ 0.2 \%$ glutaraldehyde $/ 0.04 \% \mathrm{CaCl}_{2}, \mathrm{pH} 7.4$, for $17 \mathrm{~h}$ at $4^{\circ} \mathrm{C}$, and washed in $0.1 \mathrm{M}$ phosphate buffer. Heads were embedded in $5 \%$ lowmelting point agarose and cut at $100 \mu \mathrm{m}$ on vibratome. TSA biotin system (PerkinElmer) was applied on the sections by incubating for 40 min in tyramide (1/20 in PBS) and $30 \mathrm{~min}$ in SA-HRP (1/50 in PBS) followed by an amplification of HRP signal using the ABC kit (Vector Laboratories) at room temperature. After developing with DAB for 15 min, sections were washed three times with $0.1 \mathrm{M}$ phosphate buffer. DABpositive sections in the region of antennal lobes, selected under light microscopy, were postfixed with $1 \%$ osmium tetroxide for $1 \mathrm{~h}$ at $4^{\circ} \mathrm{C}$, dehydrated in ethanol series, and embedded in Araldite using standard protocols. Blocks were cut on a Reichert Jung Ultracut E ultramicrotome at $2.5 \mu \mathrm{m}$, and semithin sections containing HRP staining in the region of interest were remounted in Araldite and sectioned to $70 \mathrm{~nm}$ ultrathin sections on an ultramicrotome Leica EM UC6. Series of ultrathin silver 
sections were collected on Formvar-coated, single-slot grids and stained with uranyl acetate $(1 \mathrm{~h})$ and lead citrate $(15 \mathrm{~min})$. Electron micrographs were produced using a Jeol 1200-EX II transmission electron microscope, collected with AnalySIS software, and analyzed with Adobe Photoshop. Reconstitution of the complete antennal lobe area from several single images was performed using Photomerge. Synapse number was calculated on images of ultrathin sections obtained from the same central area of antennal lobe in control and experimental genotypes.

Behavioral assay and odorants. Tested odorants (purity $>95 \%$ ) were from Fluka, except ethyl hexanoate from Aldrich. The olfactory response index (OI) was calculated on 5- to 7-d-old adults using a T-maze test as previously described (Acebes and Ferrús, 2001). The index indicates the number of flies trapped in the odor compartment subtracted by the number of flies trapped in the control compartment, and divided by the total number of flies. OI values range from 1 (total attraction) to -1 (total repulsion). Each data point represents the response of 250-350 individuals distributed in 10 replicates. Flies not making any choice were excluded. Tests where more than one-third of the flies did not make any choice were discarded. Flies were only tested once and all experiments were conducted in the dark at room temperature. Each odorant assay included control and experimental genotypes in a randomized sequence. Male and female adults were tested separately. Since no gender differences were observed, data are shown pooled.

Immunostaining and image analysis. Single-neuron mosaics were obtained as FRT/FLP recombinants in the construct $U A S-f r t C D 2 y^{+}$frtCD8GFP/hsflp by applying a mild heat shock $\left(37^{\circ} \mathrm{C}\right.$ during $\left.55 \mathrm{~min}\right)$ in late third-instar larvae and also early pupae stages. For whole-mount immunostainings, male brains were dissected in PBS and fixed in PLP fixative ( $2 \%$ paraformaldehyde, $0.075 \mathrm{M}$ lysine, $0.037 \mathrm{M}$ sodium phosphate) for $1 \mathrm{~h}$ at room temperature, washed in PBT (PBS $+0.5 \%$ Triton X-100), and preincubated in $90 \%$ PBT/10\% normal goat serum. Brains were incubated with primary antibodies anti-GFP (1/500, Invitrogen) and/or monoclonal antibody nc82 (1/10, Developmental Studies Hybridoma Bank) overnight at $4^{\circ} \mathrm{C}$ and secondary fluorescent antibodies Alexa 488 and Alexa 568 (Molecular Probes) for $2 \mathrm{~h}$ at room temperature. Specimens were finally mounted in Vectashield (Vector Laboratories). Confocal images were acquired on a Leica TCS-SP5. Serial optical sections $(512 \times 512$ pixels) were taken at $1 \mu \mathrm{m}$ intervals using $40 \times$ or $63 \times$ objectives. Functional-imaging procedures were as described previously (Yu et al., 2004). Each fly was stimulated with two consecutive odor presentations [benzaldehyde (BZD), isoamylacetate (IAA)], spaced $1.5 \mathrm{~min}$. Images were acquired at 5 frames/s and each frame was calculated as average of three scannings at $512 \times 512$ pixel resolution. The raw fluorescence images were first smoothed with a $5 \times 5$ Gaussian blur convolution filter and registered using ImageJ software (National Center for Biotechnology Information). Regions of interest were circumscribed and a pseudocolor image of the $\% \Delta F / F_{\mathrm{o}}$ ratio was obtained. $F_{\mathrm{o}}$ values were calculated for each pixel within the region of interest as basal fluorescence before odor application and averaged over five successive frames. The $\Delta F$ was calculated for each pixel within the region of interest as the difference between the maximum average intensity during the $3 \mathrm{~s}$ odor application for five successive frames and basal $F_{\mathrm{o}}$. To estimate the size of the ALs in the various genotypes, we used the confocal images produced by the GFPtagged synaptic marker n-synaptobrevin (UAS-syb-GFP) driven by the corresponding Gal4. Image analysis was performed using ImageJ version 1.44 (http://rsb.info.nih.gov/ij/). Pixel intensities were not used for these measurements. $Z$ sections ( $1 \mu \mathrm{m}$ ) were used to create a real image of the AL. The total AL volume was estimated as the surface occupied by each confocal section multiplied by the number of sections. The AL maximum area means the section of largest surface. The freehand selection tool, which allows drawing the perimeter of each image, was used to measure area and volume. Laser settings were maintained throughout all the genotypes analyzed.

Statistical analysis. Data represent mean \pm SEM. In behavioral experiments, statistical significance was calculated using Student's two-tailed $t$ test. Significant differences between groups were noted by asterisk: ${ }^{\star} p<$ $0.05,{ }^{* *} p<0.001,{ }^{* *} p<0.0001$. The software programs SPSS and GraphPad Instat 3 were used throughout. In the functional imaging experiments, variation among group means is significantly greater than
Table 1. Number of cells in the GH298-Gal4, krasavietz-Gal4, GH298-krasavietzGal4, and Line9-Gal4 domains and effects of expressing ChATGal80, GSK3, or PI3K $K^{\text {DN }}$ Cell number

\begin{tabular}{lr}
\hline GH298-Gal4 & \\
GH298-Gal4 & $31 \pm 1(n=5)$ \\
GH298-Gal4/ChATGal80 & $28(n=5)$ \\
GH298-Gal4/GSK3 & $30 \pm 1(n=5)$ \\
GH298-Gal4/PI3K & $32 \pm 1(n=5)$ \\
Krasavietz-Gal4 & \\
$\quad$ Krasavietz-Gal4 & $6 \pm 1(n=8)$ \\
Krasavietz-Gal4/ChATGal80 & $2(n=6)$ \\
Krasavietz-Gal4/PI3K & $6 \pm 1(n=6)$ \\
GH298-Gal4/Krasavietz-Gal4 & \\
GH298-Gal4/Krasavietz-Gal4 & $36 \pm 1(n=6)$ \\
GH298-Gal4/Krasavietz-Gal4PI3K & $37 \pm 1(n=8)$ \\
Line9-Gal4 & \\
Line9-Gal4 & $16 \pm 1(n=5)$ \\
Line9-Gal4/ChATGal80 & $12 \pm 1(n=5)$ \\
Line9-Gal4/UAS-PI3K & $15 \pm 1(n=5)$ \\
\hline
\end{tabular}

expected by chance and all data groups passed the Kolmogorov and Smirnov normality test before ANOVA.

\section{Results}

Identification of neuron types in the Gal4 domains

In the first series of experiments, we used GH298-Gal4 and krasavietz-Gal4 for expression in 30-32 and 6-8 neurons per antennal lobe, respectively (Fig. $1 A, B$, Table 1 ). The first group is reported to correspond mostly to GABA-immunopositive neurons ( $\mathrm{Ng}$ et al., 2002), while most of the second-group neurons are choline acetyl transferase (ChAT) immunopositive (Shang et al., 2007). Here, we confirmed these data by the expression of a $3.3 \mathrm{~kb}$ enhancer from the ChAT gene coupled to the Gal4 inhibitor Gal80 (Kitamoto et al., 1992) to silence the expression of ChAT cells in both groups. The test shows that $~ 90 \%$ of GH298 neurons are not cholinergic, while $66 \%$ of krasavietz neurons are cholinergic [data in agreement with Shang et al. (2007)] (Fig. $1 C, D$, Table 1$)$. The variability in cell numbers remains in the range of $5-10 \%$ both between and within individuals, which is consistent with other quantitative data on the number of axons in the antennal nerve (Acebes and Ferrus, 2001). The genetic constructs corresponding to PI3K ${ }^{\mathrm{DN}}$ and GSK3 were expressed in selected neurons using specific Gal4 drivers (Brand and Perrimon, 1993). Also, we generated single-neuron mosaics by means of FRT/FLP recombination (Nellen et al., 1996) to determine whether the morphological effects of altering the synaptogenic signaling in AL local neurons are as expected from similar manipulations in other neural centers (Martín-Peña et al., 2006). Both types of neurons, GH298 and krasavietz, reduce their branching if PI3K ${ }^{\mathrm{DN}}$ or GSK3 are overexpressed (Fig. $1 \mathrm{E}-\mathrm{H}$ ). Although these constructs have been previously used in larval motor neurons and large-field neurons of the ellipsoidal body where synapse numbers were directly counted (Martín-Peña et al., 2006), we repeated the electron microscopy study in the AL (Fig. 2). Membrane profiles of the targeted neurons were identified by the coexpression of a UAS-CD2-HRP construct. Here, as in the first original study (Watts et al., 2004), the identification of the HRP profiles is not homogeneous throughout the cell profiles for unknown reasons. Thus, we counted synapses in reference to the total length of positively identified HRP membrane. A total length of 966.8 and $1072.8 \mu \mathrm{m}$ were analyzed in the control $(n=$ 3 sections) and GSK3 ( $n=3$ sections) expressing genotypes, respectively. The data show 515 and 395 synapses in control and 

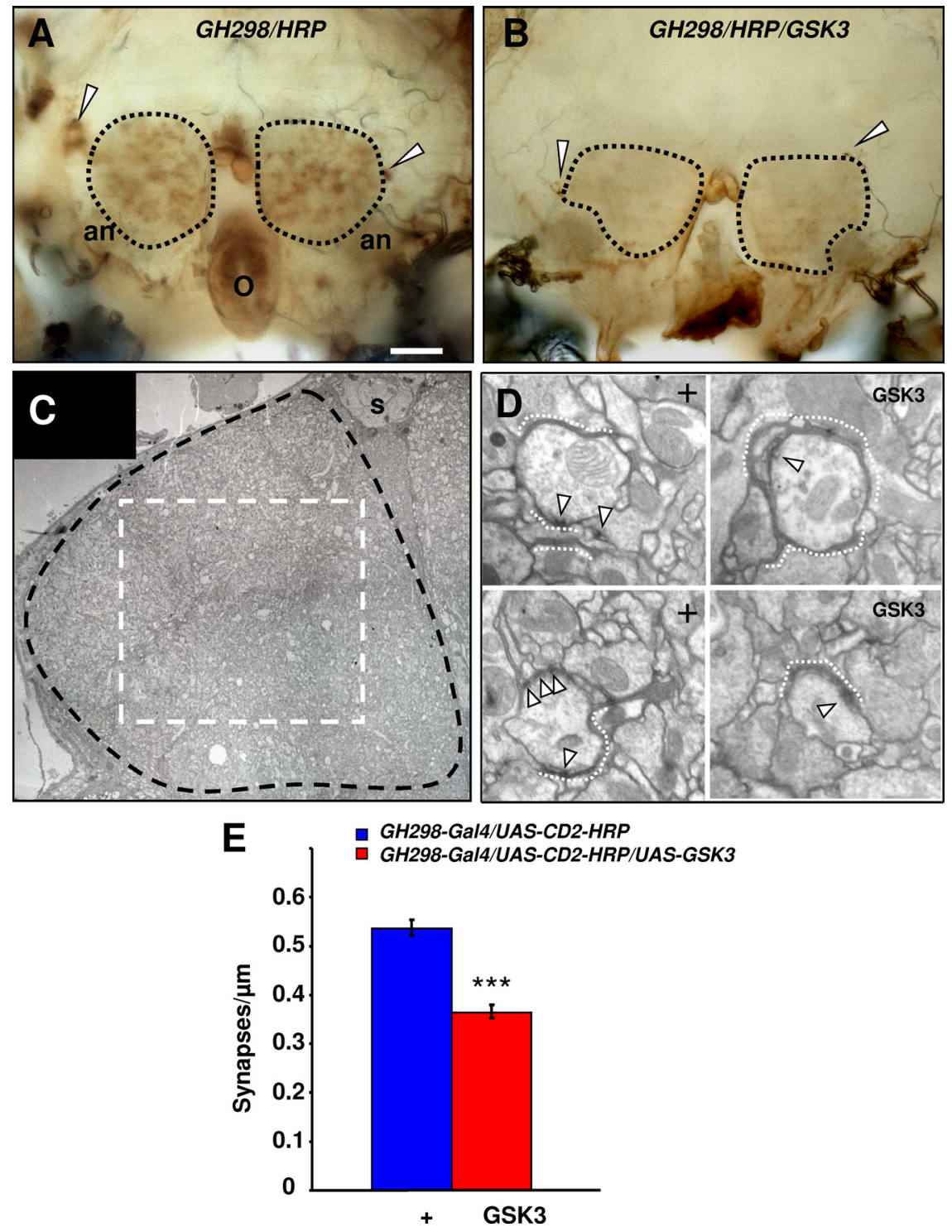

Figure 2. GSK3 expression decreases the number of synapses in antennal lobe local neurons. $A$, Frontal view of a brain agaroseembedded semithin section (genotype: GH298-Gal4 > UAS-CD2-HRP) stained for HRP. Note the brown signal in glomeruli within the antennal lobe (dashed lines) and the corresponding somata (arrows). $\boldsymbol{B}$, Effects of GSK3 coexpression in the same genotype. Note the reduced HRP signal with respect to $A$. an, Antennal nerve; 0 , oesophagus. $C$, Low-magnification TEM view showing the right antennal lobe (black dashed line) from a GSK3-expressing brain (ultrathin section from the semithin section shown in $\boldsymbol{B}$ ). The area from which synapses were counted (white dashed line) corresponds to the neuropile of the antennal lobe where somata ( $s$ ) are not present. $\boldsymbol{D}$, High-magnification TEM views from the central neuropile (white dashed area in $\boldsymbol{C}$ ) of antennal lobes in control $(+)$ and GSK3 (right two panels)-expressing brains. HRP deposits label cell profiles in dark, thus identifying GH298 neurons. Often, HRP deposits are recognized in a fraction of the cell profile (white dashed lines) (see also Watts et al., 2004). Synapses (white arrowheads) are visible on HRP-negative as well as on HRP-positive membranes, but they are counted on the latter only. E, Quantification of synapse number over total length of HRP-positive cell membranes. The GSK3-expressing brain shows a significant reduction compared to the control. ${ }^{* * *} p=0.0002$ (Student's $t$ test). Scale bar (in $\boldsymbol{A}$ ): $\boldsymbol{A}, \boldsymbol{B}, 30 \mu \mathrm{m} ; \boldsymbol{C}, 15 \mu \mathrm{m} ; \boldsymbol{D}, 1 \mu \mathrm{m}$.

GSK3 (mean \pm SEM $=0.54 \pm 0.01$ and $0.37 \pm 0.01$ respectively, $p=0.0002$, Student's $t$ test) (Fig. 2E). The synapse reduction effect is further confirmed for PI3K ${ }^{\mathrm{DN}}$ expression using the presynaptic marker synaptobrevin (supplemental Fig. S1, available at www. jneurosci.org as supplemental material). To determine possible effects of PI $3 \mathrm{~K}^{\mathrm{DN}}$ or GSK3 on cell proliferation (Leevers et al., 1996), we counted the number of neurons monitored with the $U A S-G F P^{n l s}$ reporter driven by the two Gal4 in control versus all mutant conditions. The data indicate no significant differences in cell number among all genotypes, which allows ruling out the influence of cell proliferation (Table 1).

\section{Synapse loss leads to reduced} neural activity

To evaluate the functional effects in the antennal lobe, we monitored changes in fluorescence as reported by G-CaMP after odorant stimulation (Wang et al., 2003). Upon delivery of odorant stimulus, benzaldehyde or isoamylacetate, the measured changes demonstrate that activity in the $\mathrm{AL}$ is reduced when PI3K $\mathrm{K}^{\mathrm{DN}}$ is expressed in GH298 neurons (Fig. $3 A$, B). Since the activity reporter is expressed in local interneurons, which project to most glomeruli, the images do not discriminate among individual glomeruli. They show, however, a direct proof that the genetic expression of PI3K ${ }^{\mathrm{DN}}$ has a functional consequence in these neurons. It is worth noting that the activity reduction appears more pronounced in the medial, rather than the lateral, region of the $\mathrm{AL}$ (Fig. $3 C$ ) for either odorant stimulation (compare panels $i$ versus $i i$, quantified in $i i i$, in sections $A$ and $B$ ). The control and $\mathrm{PI}^{3} \mathrm{~K}^{\mathrm{DN}}$ expressing GH298 neurons do not exhibit a preferential branching in the medial $\mathrm{AL}$; therefore, this activity difference could, perhaps, indicate a functional specialization within the branching arbor of the GH298 set of neurons. The odors used, benzaldehyde and isoamylacetate, are reported to activate mainly the glomeruli VM3 and DM6 (benzaldehyde) and DA4M, VM3, DM2, VM2, DM3, DM6, and VM5 (isoamylacetate) (Couto et al., 2005; Hallem and Carlson, 2006), all of which are located within this medial region of the AL.

\section{Synapse loss leads to odorant perception changes}

To analyze potential changes in perception, a behavioral assay is required. We measured the odorant choice index over a $10^{-7}$ to $10^{-1}(\mathrm{v} / \mathrm{v})$ concentration range for volatiles representing diverse chemical groups: BZD, ethyl hexanoate (EH), IAA, 1-hexanol (1-Hex), ethyl butyrate (EB), and propionic acid (PA). First, we addressed the role of GH298 neurons in perception. Under reduction of synapses in the GH298 domain, responses shift toward repulsion (Fig. $4 A-D$ ). The effect is independent of the method used to reduce synapse number, GSK3 or PI $3 \mathrm{~K}^{\mathrm{DN}} \mathrm{ex}-$ pression (Fig. 4D). Also, this effect is consistent among all odorants tested and throughout the range of concentrations. It should be noted, however, that the strength of the repulsive reaction is still proportional to the odorant concentration. Thus, the reduced number of synapses does not seem to affect the stimulus dosage dependence of the behavioral response.

Although the GH298 domain consists of mostly inhibitory neurons, it is contaminated with a few ChAT-expressing cells. To dissect the specific role of inhibitory versus excitatory GH298 
neurons in olfactory perception, we performed some tests in genotypes where the Gal4 repressor Gal80 is expressed in those GH298 neurons that are ChAT positive. The data show that the shift toward repulsion observed when the whole set of GH298 synapses are reduced remains evident and with the same magnitude (Fig. $4 E)$. Thus, the few excitatory neurons of the GH298 domain do not seem to contribute noticeably to the change in olfactory perception caused by the synapse loss in the whole GH298 neuron subset.

In a second series of experiments, we addressed the krasavietz domain of local interneurons. Here, the reduction of synapses yielded opposite effects to those of GH298. Odorant responses shifted toward attraction (Fig. 4F). The highest stimulus concentration still evoked repulsive reactions, although of a significantly lower magnitude than controls. Thus, as in the case of $\mathrm{GH} 298$, the stimulus dosage dependence of the behavioral responses is still preserved. Most of the krasavietz AL neurons are considered excitatory based on the expression of ChAT. However, a few AL neurons in this domain do not express the ChAT gene and, thus, could be inhibitory. To discriminate between the excitatory and nonexcitatory contributions of the krasavietz AL neurons, we tested olfaction in a genotype that prevents the Gal4 expression in the ChAT neurons specifically (Fig. $4 F$ ). In this case, the shift toward attraction previously observed is completely abolished. Thus, we concluded that the change in olfactory perception caused by synapse loss in the krasavietz domain is due to the ChATexpressing, and so presumably excitatory, neurons of this domain. The consistency between odorants and behavioral effects demonstrates that the amount of synapses in the GH298 and krasavietz neurons is critical for stimulus perception. Also the data indicate that the olfactory perception changes elicited by the reduction of synapses in the GH298 and krasavietz domains result from the contribution of inhibitory and excitatory synapses, respectively.

Another Gal4 driver has been reported under the name of krasavietz, pointing out a discrepancy with the domain of expression of the original line reported with that name (Shang et al., 2007). We obtained that second line (kindly provided by Dr. Luo) and analyzed its expression domain and olfactory perception profile under PI3K ${ }^{\mathrm{DN}}$ expression (supplemental Fig. S2, available at www.jneurosci.org as supplemental material). The morphological data on this line confirm those reported (Chou et al., 2010) (Table 1) but are clearly different from those of the original krasavietz (Shang et al., 2007) (Table 1). Consequently, we have renamed that line as Line 9 instead of krasavietz. In addition, considering the putative inhibitory nature of the Line 9 neurons, we tested olfactory perception under synapse loss in these neurons. The data show a behavioral shift toward repulsion, which is conindicate mean \pm SEM.
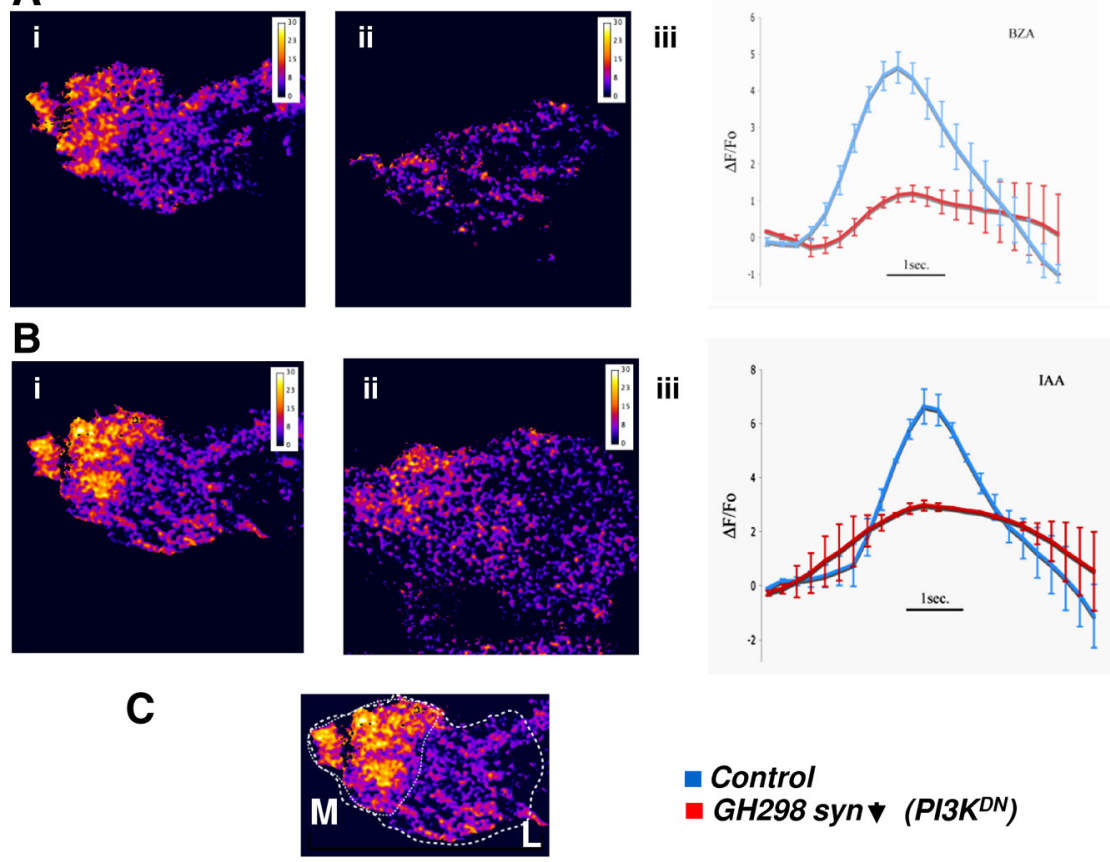

\section{- Control \\ - GH298 synt (PI3K $\left.{ }^{D N}\right)$}

Figure 3. Functional effects of reducing the number of synapses. $\boldsymbol{A}$, Visualization of neuronal activity by driving the functional reporter UAS-G-CaMP to the GH298-Gal4 domain. The percentage change in fluorescence $(\Delta F)$ relative to baseline $\left(F_{0}\right)$ is illustrated as a pseudocolor image following presentation of BZD $(\boldsymbol{A})$ or IAA $(\boldsymbol{B})$ in control (i) and UAS-PI3K ${ }^{D N}$ (ii)-expressing antennal resenting the average response from different brains at the peak of the fluorescence signal from the whole, medial (M), or Iateral (L) regions of the antennal lobe in the two genotypes. Stimulation with BZD or IAA yielded significant differences, demon-

sistent with the effect on the other inhibitory GH298 neurons (supplemental Fig. S2C,D, available at www.jneurosci.org as supplemental material). In agreement with the behavioral effects of this Line9-renamed Gal4 line, a patch-clamp study of the krasavietz-named Gal4 line shows that most of them are GABAergic (Seki et al., 2010).

\section{Odorant perception is dependent on the ratio of excitatory/inhibitory synapses}

To assay the relationship between the two types of synapses represented in the GH298 and krasavietz domains during perception, we combined both Gal4 drivers to elicit the simultaneous reduction of both subsets of synapses. In these flies, the responses along the whole range of 1-hexanol concentrations were fully coincident with those from the controls (Fig. 5). This normal perception when both set of neurons are affected was also observed with odorants of different chemical nature, IAA and EB (supplemental Fig. S3, available at 
A

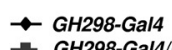

$\rightarrow$ GH298-Gal4/UAS-GSK3

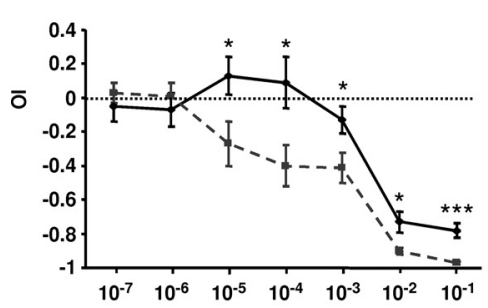

PA
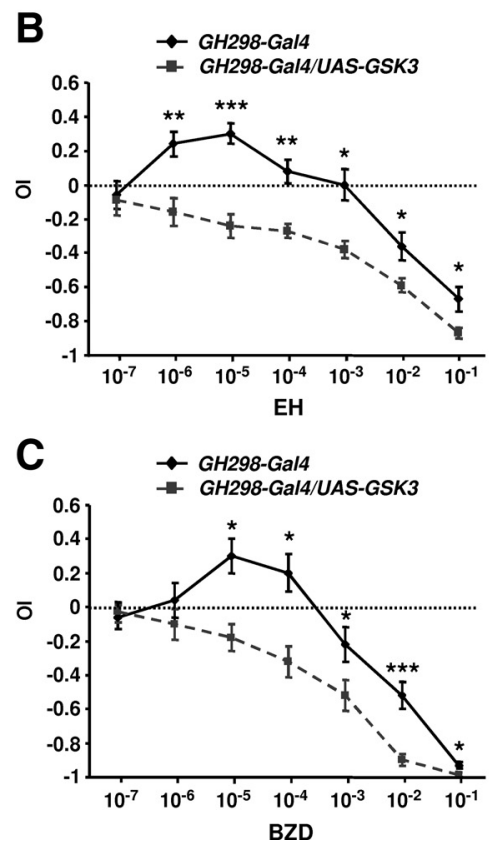

D

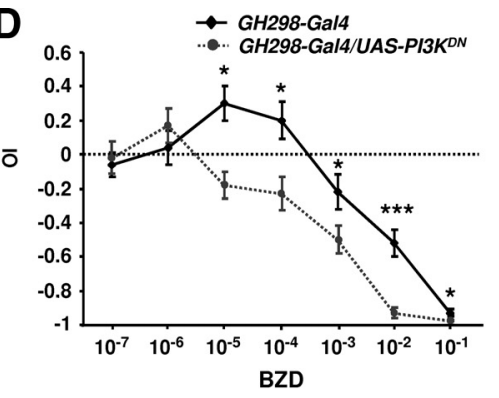

E

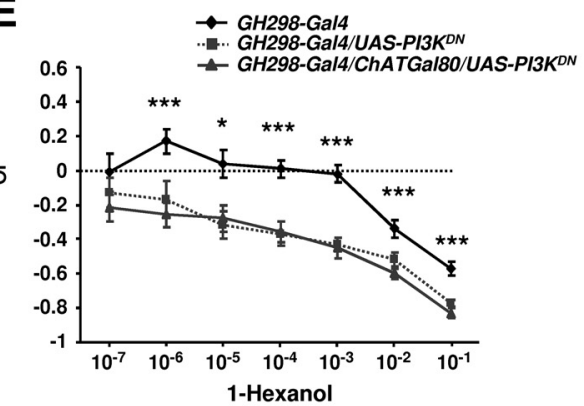

$\mathbf{F}$

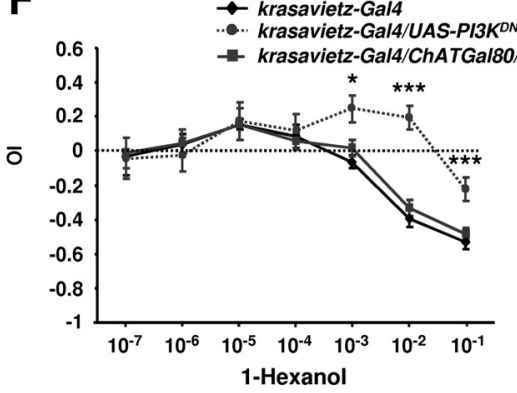

Figure 4. Behavioral effects of reducing the number of synapses. Mature adults were subject to an odorant choice test over a range of concentrations ( $\mathrm{v} / \mathrm{v}$ ) (Acebes and Ferrus, 2001; Devaud et al., 2001). The solvent, paraffin oil, was used as control odorant. 0 values were calculated on $250-350$ flies per data point and genotype. Tests were run in a randomized pattern for odorants, concentrations, and genotypes over a total period of 20 months. Each group of flies was subject to one odorant concentration choice only. No significant locomotion effects were found in any of the genotypes analyzed. $\boldsymbol{A}-\boldsymbol{C}$, When synapse reduction was elicited in the GH298 domain (genotype: GH298-Gal4> UAS-GSK3), responses to PA (A), EH (B), or BZD (C) were consistently repulsive (gray dotted lines in $\boldsymbol{A}-\boldsymbol{C}$ ) compared to $\mathrm{GH} 298$-Gal4 controls (full black lines). D, The repulsion effect was also evident (BZD) when UAS-PI3K $K^{D N}$ expression was used as a method to reduce the number of synapses. The GH298 domain is composed mostly of inhibitory neurons; it includes three that express ChAT (Table 1). However, silencing the Gal4 expression in these ChAT neurons still shows the regular perception shift toward repulsion (full gray line in $\boldsymbol{E}$ ). When synapse reduction was elicited in the krasavietz neurons (genotype: krasavietz-Gal $4>U A S-P I 3 K^{D N}$ ), responses for 1-Hex were consistently more attractive than controls $(\boldsymbol{F})$ (dotted gray line). This effect is abolished if the ChAT component of this Gal 4 domain is silenced by the repressor Gal80 (F) (full gray line). ${ }^{*} p<0.05 ;{ }^{* *} p<0.001 ;{ }^{* * *} p<0.0001$ (Student's $t$ test).

www.jneurosci.org as supplemental material), indicating that the effect is stimulus independent.

AL local interneurons exhibit a variety of projection profiles, and this heterogeneity is likely to represent an equivalent functional diversity (Tanaka et al., 2009; Chou et al., 2010). Thus, we performed a third series of experiments using additional Gal4 drivers to assay the behavioral effects of synapse loss in other subsets of neurons. Drivers $L N 1$ and $L N 2$ are expressed in about 18 and 37 local AL interneurons, respectively, which seem to be inhibitory based on the expression of GABA and the lack of expression of ChAT (Okada et al., 2009). We expressed GSK3 in these two domains (Fig. 6) and assayed the perception of EB and IAA (Fig. 7). In the case of $L N 1$, synapse loss had no detectable effect in odorant perception (Fig. 7A,B). The simultaneous loss of synapses in the LN1 and the krasavietz domains exhibits the typical effect due to krasavietz alone, demonstrating that these two sets of neurons are functionally independent from each other for the perception of the odorants tested. By contrast, in the case of $L N 2$, perception of $E B$ and IAA is affected in the same way as in the case of GH298 and Line9 neurons, shifting responses toward repulsion (Fig. 7C,D). Most interesting, the simultaneous alteration of LN2 and krasavietz neurons restores normal perception, reproducing the same phenomenon as in the GH298/ krasavietz combination. Together, these data indicate that normal perception depends on the local excitation/inhibition ratio rather than the absolute number of either type of synapses. This conclusion, however, does not preclude that subsets of AL neurons might have specific roles in perception or define the domains where the excitation/inhibition ratio will be relevant for the perception of a given odorant.

\section{Odorant perception effects do not result from major developmental plasticity changes of local AL neurons} Since the genetic drivers used in these experiments are chronically active, it could be argued that the behavioral effects may result from connectivity rearrangements during development. To investigate this possibility, we replicated some experiments with genotypes that allow activation of the Gal4 drivers at the desired time in the adult fly using a temperaturesensitive form of the Gal4 inhibitor, Gal80 ${ }^{t s}$. Equivalent experiments on larval motor neurons and adult interneurons of the ellipsoidal body had shown that synapses can be built or dismantled with a lifetime of approximately $24 \mathrm{~h}$ (MartínPeña et al., 2006; Owald and Sigrist, 2009), a lifetime that seems to be conserved in vertebrates as well (Aloy et al., 2006). Here, we allowed $6 \mathrm{~d}$ for the activated Gal4 to exert its effect. The data show that the effects of the time-controlled activation of the GH298 and krasavietz neuron subsets are the same as those observed with the chronic Gal4 activation (Fig. 8). Thus, we conclude that the perception effects result not from a rearrangement of the circuit during development, but from a cell autonomous change where the genetic constructs are driven.

\section{Odorant perception is not modified by synapse loss in the mushroom body neurons}

Since the expression domain of krasavietz-Gal4 includes some neurons projecting to the mushroom bodies (MB) (Fig. 9) and this brain center is implicated in odorant processing (Murthy et al., 2008), we addressed its possible role in the phenomenon described here. To that end, we measured olfactory perception under two types of genetic conditions: silencing the MB Kenyon cells (MBKCs) of the krasavietz domain, and reducing the number of synapses in most MBKC neurons. To silence MBKC neu- 

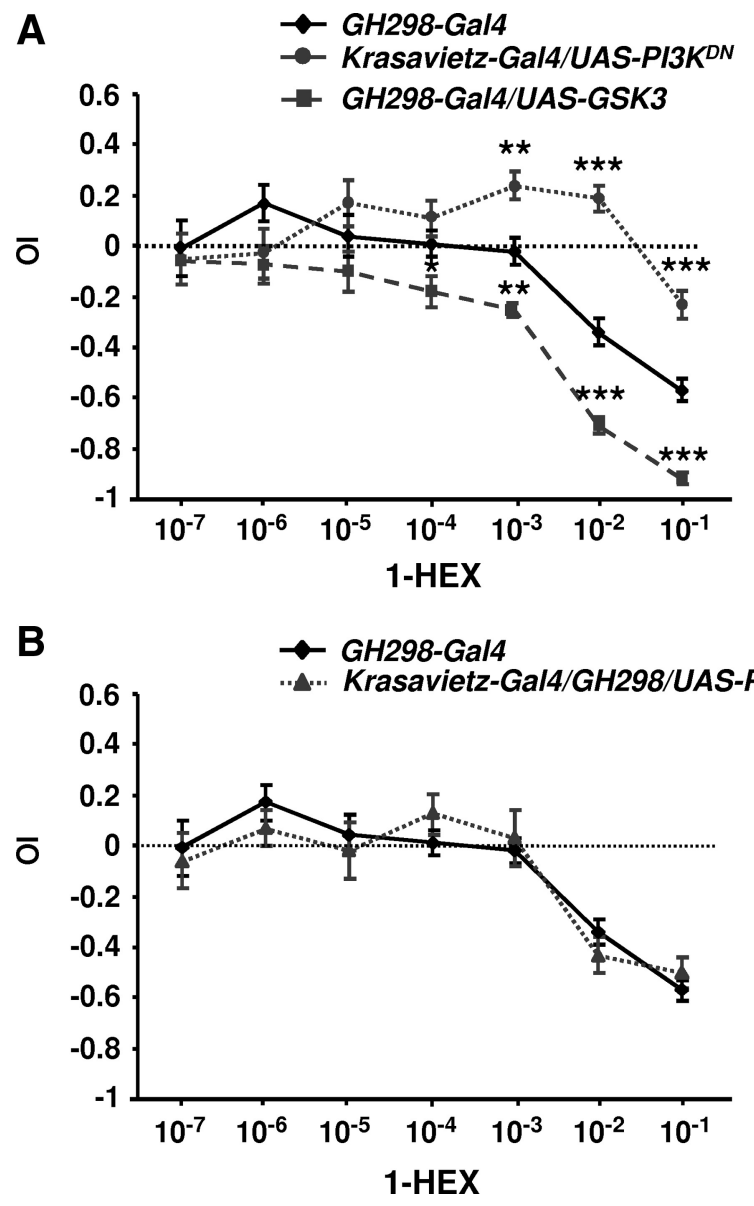

Figure 5. Normal perception with simultaneous synapse reduction. $A$, Comparison of olfactory indexes for 1-Hex when synapse reduction is elicited in each Gal4 domain separately (gray dotted lines). $\boldsymbol{B}$, When synapse reduction was elicited in both subsets of neurons (genotype: GH298-Gal4, krasavietz-Gal4 $>$ UAS-PI3K ${ }^{D N}$ ), the olfactory index returned to the normal profile (gray dotted line). The return to normal perception when GH298 and krasavietz neurons are simultaneously addressed is also reproduced with IAA and EB odorants (supplemental Fig. S3, available at www.jneurosci.org as supplemental material).

rons in the krasavietz domain, we used the construct MBGal80 (Krashes et al., 2007), which expresses the Gal4 repressor in intrinsic $\mathrm{MB}(\mathrm{KC})$ neurons only. In this genotype, the reduction of synapses still leads to the increase of olfactory indexes when tested for 1-Hexanol (Fig. 10A).

A direct approach to the possible role of $\mathrm{MB}$ neurons in the odorant perception changes described above consists in driving GSK3 to the domain of 201Y-Gal4. As in other brain centers, the upregulation of GSK3 causes a substantial reduction of neural branching in its $\mathrm{MB}$ domain (Fig. $10 B-E$ ). However, the two odorant perception profiles tested were indistinguishable from controls (Fig. 10F, G). Likewise, driving PI3K ${ }^{\mathrm{DN}}$ to the MB247 domain resulted in no change in odorant perception despite the obvious morphological changes (supplemental Fig. S4, available at www.jneurosci.org as supplemental material).

Finally, a set of krasavietz neurons belong to extrinsic $\mathrm{MB}$ neurons, within the PPL1 cluster, and some of them express tyrosine hydroxylase $(\mathrm{TH})$ and are hence putatively dopaminergic (Krashes et al., 2009; Mao and Davis, 2009). To assay the role of these neurons in the perception changes elicited by PI3K ${ }^{\mathrm{DN}}$, we made use of a TH-Gal80 construct to silence the krasavietz-Gal4 in these cells. This silencing did not prevent the change in olfactory perception that is characteristic of krasavietz-Gal4 (Fig. 11).
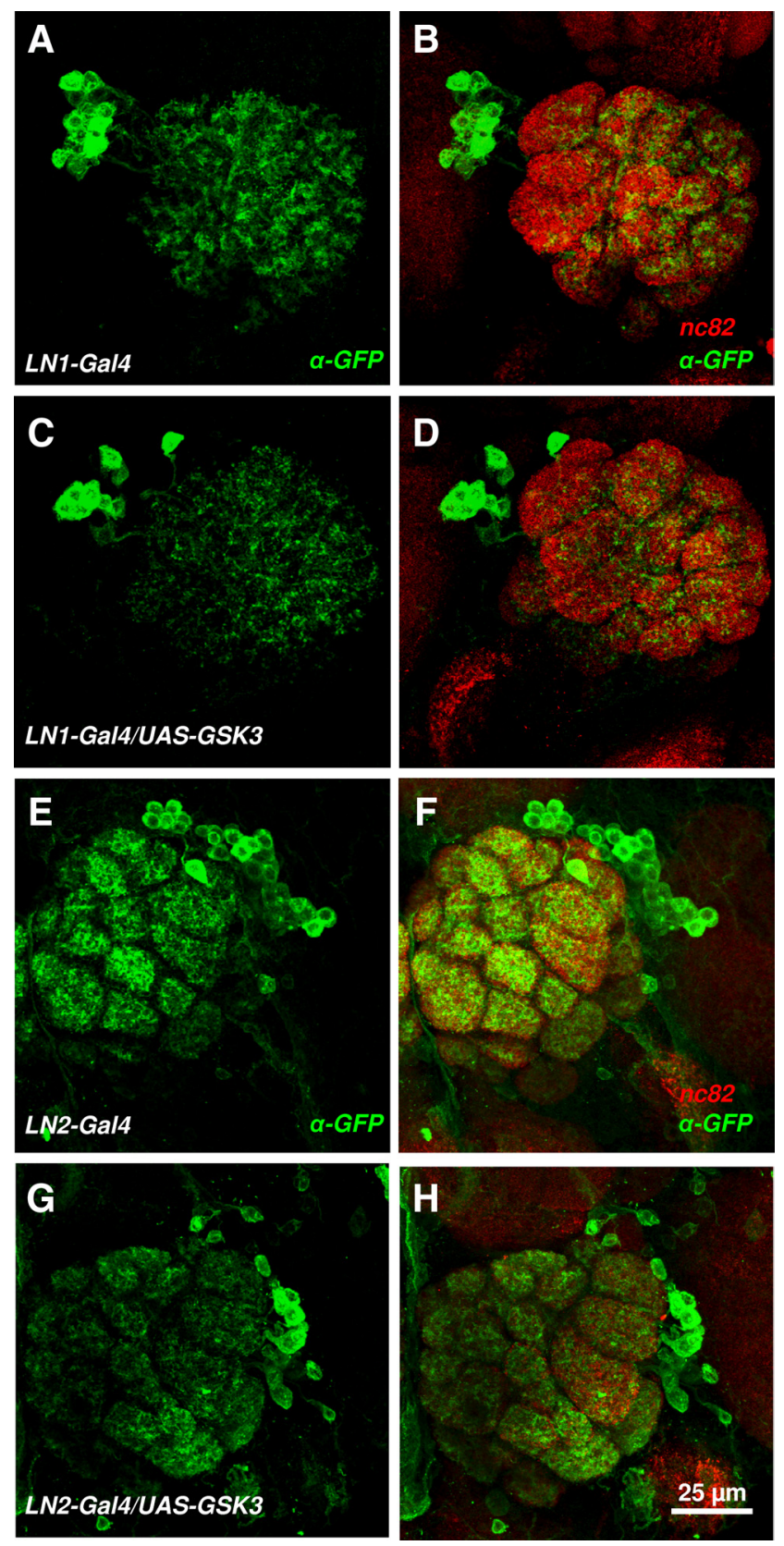

Figure 6. Expression domains of LN1-Gal4 and LN2-Gal4. Each panel shows representative confocal sections of each genotype visualized with a CD8-GFP reporter and counterstained with the nc 82 monoclonal antibody (anti-Bruchpilot). $\boldsymbol{A}, \boldsymbol{B}, \mathrm{LN} 1$ domain. $\boldsymbol{C}, \boldsymbol{D}$, Effect of driving GSK3. $\boldsymbol{E}-\boldsymbol{H}$, Equivalent genotypes using the LN2 driver. Note the reduction in branching and GFP signal intensity due to GSK3 expression.

Since the TH-Gal80 still leaves some PPL1 neurons of the krasavietz domain unaffected, we cannot formally rule out their putative contribution to the perception changes. However, we can conclude that the dopaminergic neurons of the krasavietz domain do not play a detectable role in the phenomena described here.

\section{Discussion}

The data reported here demonstrate that reduced synapse number (Fig. 2) and activity (Fig. 3) in olfactory local interneurons lead to sensory perception changes to a number of odorants (Fig. 4). The quantitative measurements based on direct synapse counts along HRP-tagged membrane profiles corresponding to GH298 neurons, the image-based expression of the synaptic 
A
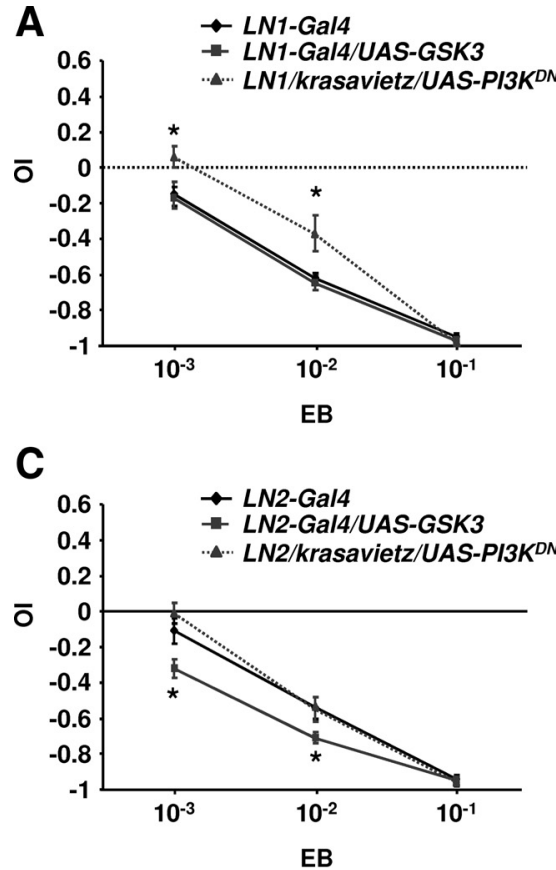
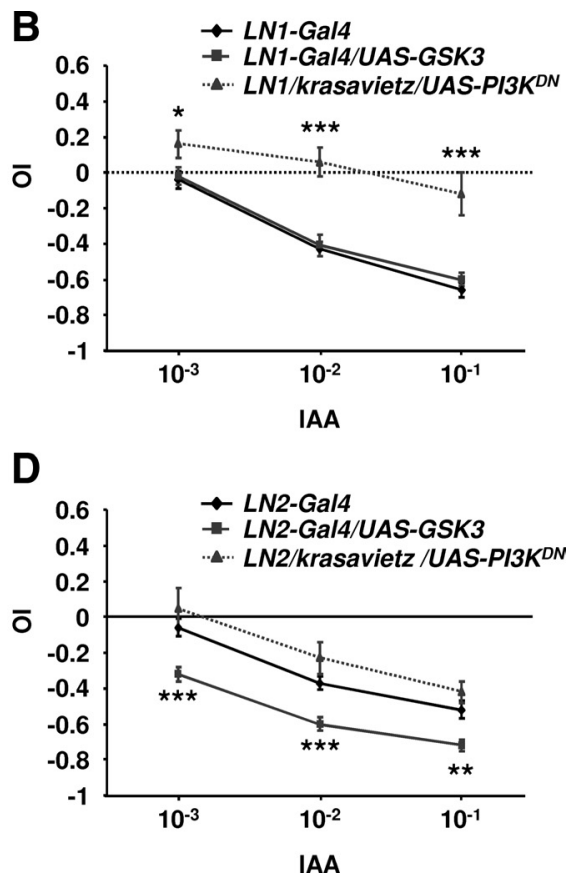

Figure 7. Olfactory perception effects in LN1 and LN2 neurons. Driving GSK3 to LN1 neurons shows no perception effect for either EB or IAA odorants (black and gray lines in $\boldsymbol{A}, \boldsymbol{B}$ ). Also, the regular effect due to krasavietz neurons is still detected when both Gal4 domains are simultaneously addressed (gray dotted lines in $\boldsymbol{A}, \boldsymbol{B}$ ). However, when LN2 neurons are used, the perception response to both odorants shifts toward repulsion (gray lines in $\mathbf{C}, \mathbf{D}$ ). This effect is akin to that of $G H 298$ neurons and consistent with the inhibitory nature of $L N 2$ cells. Furthermore, the return to normal perception is reproduced when LN2 and krasavietz neurons are affected simultaneously by $\mathrm{PI} 3 \mathrm{~K}^{\mathrm{DN}}$ (gray dotted lines in $\boldsymbol{C}, \boldsymbol{D}$ ).

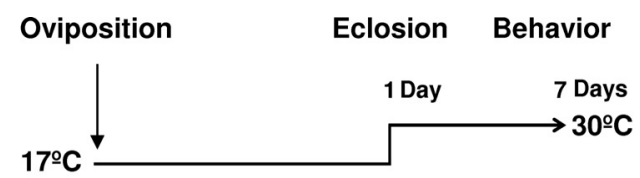

A

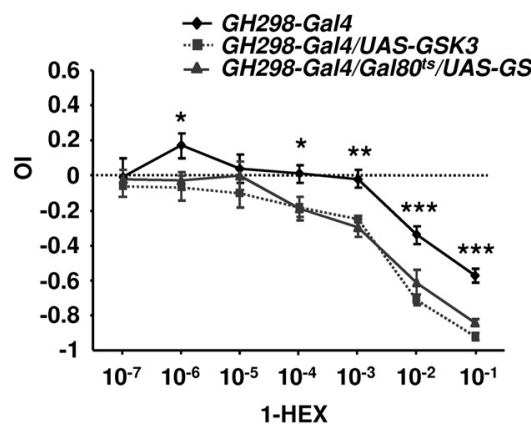

\section{B}

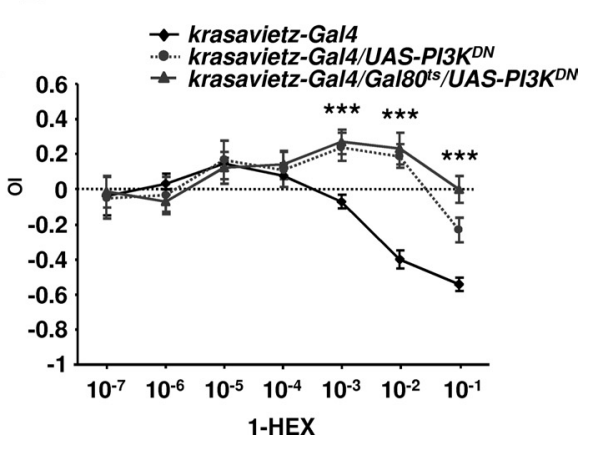

Figure 8. Temporal control of Gal4 expression. To analyze putative effects of synapse reduction during development, crosses were maintained at $17^{\circ} \mathrm{C}$ to keep the Gal 4 inhibitor $G$ al $80^{t s}$ active. At the first day of adult life, cultures were shifted to $30^{\circ} \mathrm{C}$, which inactivates the inhibitor, until the seventh day, when the olfactory tests were performed. $\boldsymbol{A}$, Effects of driving GSK3 in the GH298-Ga/4 domain compared to the data obtained without the Gal80 $0^{t 5}$ inhibitor. B, Equivalent data for the krasavietz-Gal4 domain. In both subsets of neurons, the effects toward repulsion (gray lines in $\boldsymbol{A}$ ) and attraction (gray lines in $\boldsymbol{B}$ ) are reproduced.

marker n-synaptobrevin, the functional effects in calcium imaging, and the resulting behavioral changes are all consistent with the synapse loss. We conclude that a causal effect of synapse reduction in the AL local interneurons on olfactory perception can be established. The direction of the perceptual change, however, is dependent on the type of neurons affected. When the inhibitory component is reduced, in GH298, LN2, and Line 9 neu- rons, perception becomes more repulsive, possibly because the intensity of each stimulus magnitude has become subjectively higher than normal. Likewise, when the excitatory synapses of the krasavietz domain are reduced, perception becomes more attractive, possibly because the stimulus is now subjectively lower. The two directions of perception changes are consistent with each other and reproduced in various genotypes and odorants. Also, the effects are consistent along a range of concentrations, indicating that the perception shift is independent from the stimulus magnitude. It may seem surprising that the extent of the perception change is about the same in both subsets of neurons, GH298, LN2, and Line 9 on the one hand and krasavietz on the other, given the difference in the number of neurons between these two groups (16-37 vs 7, respectively). However, one should realize that krasavietz neurons exhibit a branching arbor that is denser than that from the other group (compare panels $E$ and $G$ in Fig. 1). Thus, the behavioral consequences of the genetic modifications used here appear to depend on the number of synapses affected rather than the number of cells.

This study is focused on the behavioral effects of activity changes in local interneurons. An electrophysiological correlate would be desirable to breach the gap between the cellular activity and behavior. A recent study (Seki et al., 2010) provides a systematic analysis of the firing properties of local interneurons showing at least two main firing patterns: bursting and tonic. While this is a key step to understand the computations within the AL that, eventually, lead to behavior, the current information still falls short of allowing meaningful reasoning on the correspondence between local interneuron firing properties and emergence of behavior. Nevertheless, the available data on the morphology (Chou et al., 2010), firing properties (Seki et al., 2010), and behavior effects after genetic modification (this study) allow us to conclude that local interneurons exhibit functional specializations that were unsuspected hereto. For example, LN1 neurons appear insensitive to the GSK3-induced changes in activity for EB and IAA perception (Fig. 7).

Among the relevant cell types to be addressed, the projection neurons (PNs) represent the key to understand, at least, the output from the genetically modified AL. The perception effects reported here imply a convergence of excitatory and inhibitory signaling upon PNs. In these neurons, the excitation/inhibition ratio would be evaluated to generate a particular output message 
toward higher brain centers, mainly the lateral horn. A thorough electrophysiological study has recently shown that eLNs can exert a dual effect on PNs, direct excitation and indirect inhibition (Yaksi and Wilson, 2010). The indirect effect seems mediated through the bidirectional signaling between eLNs and iLNs, both of which make functional contacts with PNs. Independent from the chemical synapses, $\mathrm{LN}-\mathrm{PN}$ and $\mathrm{PN}-\mathrm{PN}$ signaling is also mediated by electrical coupling (Yaksi and Wilson, 2010). Whether or not this type of gap junction is affected by the genetic manipulations used here is not known. However, since the silencing of chemical synapses does not prevent the spread of, presumably electric, signaling (Root et al., 2007), while the absence of gap junctions in the shak $B^{2}$ mutant does interfere with the development of chemical synapses (Yaksi and Wilson, 2010), it is likely that the effects on chemical synapses elicited here by GSK3/PI3K ${ }^{\mathrm{DN}}$ will have little or no effect on electrical synapses. In addition to these functional studies, quantitative ultrastructural analyses will be required to settle the synaptic array between the different AL neurons and to understand the relationships between the computational properties within the AL and behavior.

The total number of local interneurons is estimated to be around 100 ipsilateral and 100 bilateral (Stocker et al., 1997; Chou et al., 2010), and the subsets used here, GH298, krasavietz, LN1, LN2, and Line9, should represent more than half of them, because these domains are largely not overlapping, if at all (Okada et al., 2009; Chou et al., 2010; our unpublished data). However, it is still formally possible that additional cells could contribute to odorant perception and information building in the AL (for a review, see Masse et al., 2009). Out of the non-AL neurons present in the krasavietz domain, we have reasonably eliminated the possible role of the intrinsic mushroom body, Kenyon cells, and TH-expressing neurons in the observed phenotypes.

One class of neurons that has not been investigated here is the neurosecretory type. Their number is small, 5-8 in the case of tachykinin-expressing cells, but their projections are quite profuse across all glomeruli (Winther et al., 2003). Also, electron microscopy images of the Drosophila AL show abundant cell terminal profiles containing large dense-core vesicles (our unpublished results). The functional role of neuropeptides in olfaction is documented to some extent (Nässel, 2002). For instance, deficits in tachykinin expression in the CNS reduce olfactory indexes (Winther et al., 2006). Also, tachykinin receptors are expressed in antennal olfactory receptor neurons, and interfering with their expression changes olfactory perception in a way similar to what we have observed here addressing local interneurons, albeit only at the highest stimulus concentration (Ignell et al., 2009). Sero-
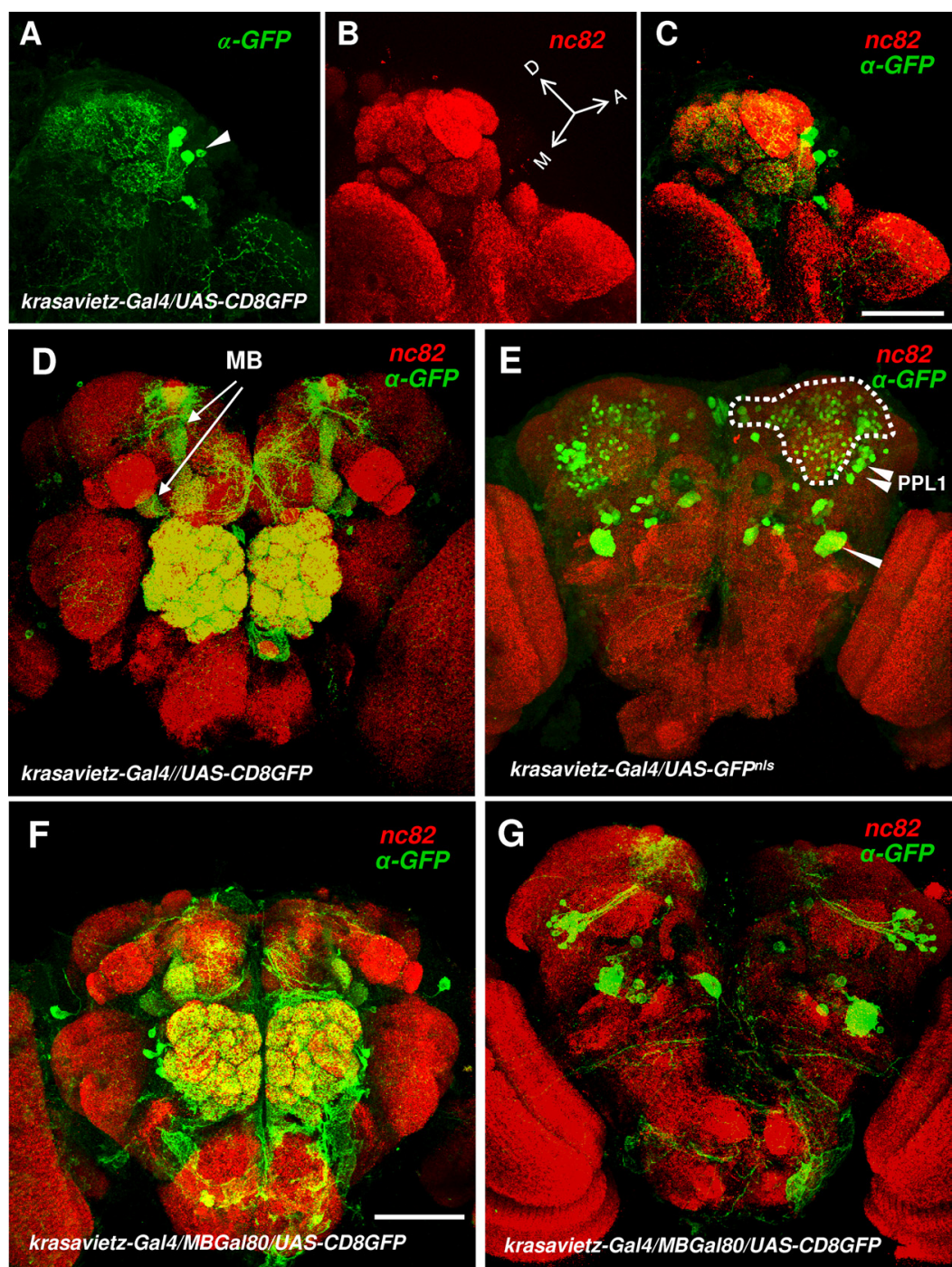

Figure 9. The krasavietz expression domain within and outside the AL. $\boldsymbol{A}-\boldsymbol{C}$, Neurons projecting to the AL visualized by the CD8-GFP reporter and counterstained with the nc82 monoclonal antibody to reveal all brain synapses. Axial coordinates in $B$ indicate anterior (A), dorsal (D), and medial (M). Under this slightly tilted orientation, the number and position of the krasavietz view of a brain expressing the nuclear GFP marker counterstained with nc 82 antibody. Mushroom body intrinsic and extrinsic (arrowheads) somata can be identified. Some krasavietz members of the PPL1 cluster (small arrow heads) are TH-expressing cells (see Results). The repressor $M B$-Gal80 is used here as the criterion to classify the krasavietz neurons as intrinsic or extrinsic to the $M B(\boldsymbol{F}, \boldsymbol{G})$. Scale bars: $\boldsymbol{A}-\boldsymbol{C}$ (in $\boldsymbol{C}), 50 \mu \mathrm{m} ; \boldsymbol{D}-\boldsymbol{G}$ (in $\boldsymbol{F}$ ), $75 \mu \mathrm{m}$.

tonin is reported to enhance the responses of inhibitory local interneurons in Drosophila (Dacks et al., 2009). The role of neuropeptides in olfaction is thought to be related to long-term conditioning of the cellular system as inferred from data in other invertebrates (Hunt, 2007; Nachman et al., 2009). The fact that perception changes can be detected in the genotypes analyzed here, even though neurosecretory cells have not been genetically modified, suggests that the role of peptides is more subtle, and perhaps longer lasting, than that caused by synapse loss elicited by GSK3 or PI3K ${ }^{\mathrm{DN}}$ expression in the five subsets of local interneurons.

Since the number of cells is not modified in the genotypes studied here, it may seem surprising that the system did not develop plastic changes that would have compensated for the synapse reduction, maintaining perception as normal. In contrast, the results show that relatively minor changes in the number of 



\section{$\mathbf{F}$}

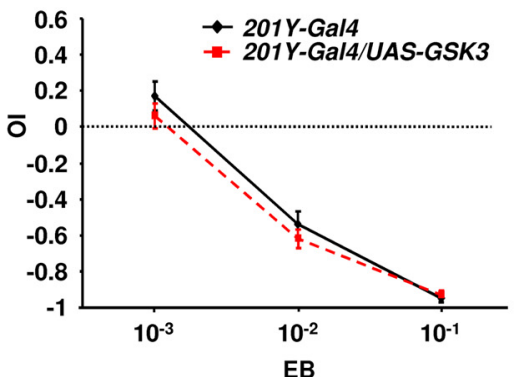

G

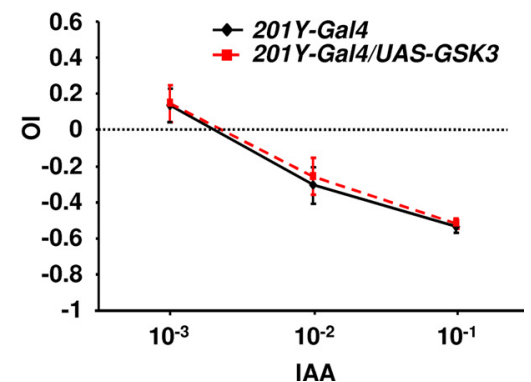

Figure 10. The mushroom body Kenyon cells of the krasavietz domain are not involved in olfactory perception changes. $\boldsymbol{A}$, Silencing the MB component of krasavietz domain leaves the perception change unaffected. $\boldsymbol{B}-\boldsymbol{E}$, The expression of GSK3 in the MB domain of $201 Y$-Gal4 causes the expected morphological effects as viewed in the peduncles $(\boldsymbol{B}, \boldsymbol{C})$ or the calyx neurites $(\boldsymbol{D}, \boldsymbol{E}) . \boldsymbol{F}, \boldsymbol{G}$, The olfactory perception to EB $(\boldsymbol{F})$ and IAA $(\boldsymbol{G})$, however, remains normal. The same result is observed with the MB247-Gal4 (supplemental Fig. S4, available at www.jneurosci.org as supplemental material).

synapses manifest in sensory changes when perception is quantitatively analyzed. It could be argued that this lack of functional redundancy in an insect brain cannot be translated to vertebrate brains, where redundancy is thought to be a hallmark (Shepherd, 1993; Barlow, 2001; Mueller and Wullimann, 2009). However, increasing evidence indicates that olfactory deficits are among the first symptoms of Parkinson, Alzheimer, and other neurodegenerative diseases (Doty, 2009; Lees et al., 2009). Clearly, normalized sensory tests and longitudinal studies are needed to further evaluate the possible correlations between subtle changes in olfactory perception and the final onset of a neurodegenerative disease.

Considering all data together, it seems that the ratio of inhibitory to excitatory synapses, rather than the absolute number, established by the antennal lobe local interneurons determines the quantitative component of perception to most, if not all, odorants. The actual value of this ratio cannot be determined because it is not possible to manipulate the complete subsets of inhibitory and excitatory neurons at the same time. Also, it is plausible that the ratio might differ between brain centers and tasks. Nevertheless, the concept of synapse ratio as the basis of normal perception proposed here may explain the similar behavioral performance of individuals with disparate brain sizes and, thus, cell numbers. Extending the present data beyond Drosophila, these results support the biological relevance of the synapse loss observed in association with certain pathological conditions (Daskalakis et al., 2007). The severity of schizophrenia might seem disproportionate to the minimal ( $\sim 16 \%)$ inhibitory synapse loss reported in patients (Benes and Berretta, 2001). However, in the fly's olfactory glomeruli, a similar percentage reduction of inhibitory synapses has drastic effects on perception that would have passed unnoticed unless quantified in a behavioral test. The overt general behavior of the experimental flies was normal. Yet, the ethological significance of these perception changes must be considerable, as indicated, for example, by the conversion of 1-hexanol (green banana) normal responses from very repulsive to clearly attractive depending on which synapse subset is affected. If the phenomenon described here is general to all species, it would lend support to therapeutical strategies based on the restoration of the synaptic ratio to recover normal cognition (Fernandez et al., 2007).

\section{References}

Acebes A, Ferrús A (2001) Increasing the number of synapses modifies olfactory perception in Drosophila. J Neurosci 21:6264-6273.

Aloy EM, Weinmann O, Pot C, Kasper H, Dodd DA, Rülicke T, Rossi F, Schwab ME (2006) Synaptic destabilization by neuronal Nogo-A. Brain Cell Biol 35:137-156.

Arevian AC, Kapoor V, Urban NN (2008) Activity-dependent gating of lateral inhibition in the mouse olfactory bulb. Nat Neurosci 11:80-87.

Barlow H (2001) Redundancy reduction revisited. Network 12:241-253.

Ben-Ari Y (2006) Seizures beget seizures: the quest for GABA as a key player. Crit Rev Neurobiol 18:135-144.

Benes FM, Berretta S (2001) GABAergic interneurons: implications for understanding schizophrenia and bipolar disorder. Neuropsychopharmacology 25:1-27.

Brand AH, Perrimon N (1993) Targeted gene expression as a means of altering cell fates and generating dominant phenotypes. Development 118:401-415.

Chou YH, Spletter ML, Yaksi E, Leong JC, Wilson RI, Luo L (2010) Diversity and wiring variability of olfactory local interneurons in the Drosophila antennal lobe. Nat Neurosci 13:439-449.

Couto A, Alenius M, Dickson BJ (2005) Molecular, anatomical, and functional organization of the Drosophila olfactory system. Curr Biol 15:1535-1547. 
Dacks AM, Green DS, Root CM, Nighorn AJ, Wang JW (2009) Serotonin modulates olfactory processing in the antennal lobe of Drosophila. J Neurogenet 23:366-377.

Daskalakis ZJ, Fitzgerald PB, Christensen BK (2007) The role of cortical inhibition in the pathophysiology and treatment of schizophrenia. Brain Res Rev 56:427-442.

Devaud JM, Acebes A, Ferrús A (2001) Odor exposure causes central adaptation and morphological changes in selected olfactory glomeruli in Drosophila. J Neurosci 21:6274-6282.

Doty RL (2009) The olfactory system and its disorders. Semin Neurol 29:74-81.

Dubnau J, Chiang AS, Grady L, Barditch J, Gossweiler S, McNeil J, Smith P, Buldoc F, Scott R, Certa U, Broger C, Tully T (2003) The staufen/pumilio pathway is involved in Drosophila long-term memory. Curr Biol 13:286-296.

Dudek FE, Sutula TP (2007) Epileptogenesis in the dentate gyrus: a critical perspective. Prog Brain Res 163:755-773.

Fernandez F, Morishita W, Zuniga E, Nguyen J, Blank M, Malenka RC, Garner CC (2007) Pharmacotherapy for cognitive impairment in a mouse model of Down syndrome. Nat Neurosci 10:411-413.

Franco B, Bogdanik L, Bobinnec Y, Debec A, Bockaert J, Parmentier ML, Grau Y (2004) Shaggy, the homolog of glycogen synthase kinase 3, controls neuromuscular junction growth in Drosophila. J Neurosci 24:65736577.

Gan WB, Kwon E, Feng G, Sanes JR, Lichtman JW (2003) Synaptic dynamism measured over minutes to months: age-dependent decline in an autonomic ganglion. Nat Neurosci 6:956-960.

Geinisman Y, Ganeshina O, Yoshida R, Berry RW, Disterhoft JF, Gallagher M (2004) Aging, spatial learning, and total synapse number in the rat $\mathrm{CA} 1$ stratum radiatum. Neurobiol $\mathrm{Ag}$ ing 25:407-416.

Hallem EA, Carlson JR (2006) Coding of odors by a receptor repertoire. Cell 125:143-160.

Helton TD, Otsuka T, Lee MC, Mu Y, Ehlers MD (2008) Pruning and loss of excitatory synapses by the parkin ubiquitin ligase. Proc Natl Acad Sci U S A 105:19492-19497.

Hooper C, Killick R, Lovestone S (2008) The GSK3 hypothesis of Alzheimer's disease. J Neurochem 104:1433-1439.

Huang J, Zhang W, Qiao W, Hu A, Wang Z (2010) Functional connectivity and selective odor responses of excitatory local interneurons in Drosophila antennal lobe. Neuron 67:1021-1033.

Hunt GJ (2007) Flight and fight: a comparative view of the neurophysiology and genetics of honey bee defensive behavior. J Insect Physiol 53:399-410.

Ignell R, Root CM, Birse RT, Wang JW, Nässel DR, Winther AM (2009) Presynaptic peptidergic modulation of olfactory receptor neurons in Drosophila. Proc Natl Acad Sci U S A 106:13070-13075.

Keller A, Sweeney ST, Zars T, O'Kane CJ, Heisenberg M (2002) Targeted expression of tetanus neurotoxin interferes with behavioral responses to sensory input in Drosophila. J Neurobiol 50:221-233.

Kitamoto T (2001) Conditional modification of behavior in Drosophila by targeted expression of a temperature-sensitive shibire allele in defined neurons. J Neurobiol 47:81-92.

Kitamoto T, Ikeda K, Salvaterra PM (1992) Analysis of cis-regulatory elements in the $5^{\prime}$ flanking region of the Drosophila melanogaster choline acetyltransferase gene. J Neurosci 12:1628-1639.

Kraft R, Levine RB, Restifo LL (1998) The steroid hormone 20- hydroxyecdysone enhances neurite growth of Drosophila mushroom body neurons isolated during metamorphosis. J Neurosci 18:8886-8899. Krashes MJ, Keene AC, Leung B, Armstrong JD, Waddell S (2007) Sequential use of mushroom body neuron subsets during Drosophila odor memory processing. Neuron 53:103-115.

Krashes MJ, DasGupta S, Vreede A, White B, Armstrong JD, Waddell S (2009) A neural circuit mechanism integrating motivational state with memory expression in Drosophila. Cell 139:416-427.

Lees AJ, Hardy J, Revesz T (2009) Parkinson's disease. Lancet 373:2055-2066

Leevers SJ, Weinkove D, MacDougall LK, Hafen E, Waterfield MD (1996) The Drosophila phosphoinositide 3-kinase Dp110 promotes cell growth. EMBO J 15:6584-6594.

Lin DY, Shea SD, Katz LC (2006) Representation of natural stimuli in the rodent main olfactory bulb. Neuron 50:937-949.

Mao Z, Davis RL (2009) Eight different types of dopaminergic neurons innervate the Drosophila mushroom body neuropil: anatomical and physiological heterogeneity. Front Neural Circuits 3:5.

Martín-Peña A, Acebes A, Rodríguez JR, Sorribes A, de Polavieja GG, Fernández-Fúnez P, Ferrús A (2006) Age-independent synaptogenesis by phosphoinositide 3 kinase. J Neurosci 26:10199-10208. 
Masse NY, Turner GC, Jefferis GS (2009) Olfactory information processing in Drosophila. Curr Biol 19:R700-R713.

McGuire SE, Le PT, Osborn AJ, Matsumoto K, Davis RL (2003) Spatiotemporal rescue of memory dysfunction in Drosophila. Science 302:17651768.

Mueller T, Wullimann MF (2009) An evolutionary interpretation of teleostean forebrain anatomy. Brain Behav Evol 74:30-42.

Murthy M, Fiete I, Laurent G (2008) Testing odor response stereotypy in the Drosophila mushroom body. Neuron 59:1009-1023.

Nachman RJ, Pietrantonio PV, Coast GM (2009) Toward the development of novel pest management agents based upon insect kinin neuropeptide analogues. Ann N Y Acad Sci 1163:251-261.

Nässel DR (2002) Neuropeptides in the nervous system of Drosophila and other insects: multiple roles as neuromodulators and neurohormones. Prog Neurobiol 68:1-84.

Nellen D, Burke R, Struhl G, Basler K (1996) Direct and long-range action of a DPP morphogen gradient. Cell 85:357-368.

Ng M, Roorda RD, Lima SQ, Zemelman BV, Morcillo P, Miesenböck G (2002) Transmission of olfactory information between three populations of neurons in the antennal lobe of the fly. Neuron 36:463-474.

Okada R, Awasaki T, Ito K (2009) Gamma-aminobutyric acid (GABA)mediated neural connections in the Drosophila antennal lobe. J Comp Neurol 514:74-91.

Olsen SR, Wilson RI (2008) Lateral presynaptic inhibition mediates gain control in an olfactory circuit. Nature 452:956-960.

Olsen SR, Bhandawat V, Wilson RI (2007) Excitatory interactions between olfactory processing channels in the Drosophila antennal lobe. Neuron 54:89-103.

Owald D, Sigrist SJ (2009) Assembling the presynaptic active zone. Curr Opin Neurobiol 19:311-318.

Root CM, Semmelhack JL, Wong AM, Flores J, Wang JW (2007) Propagation of olfactory information in Drosophila. Proc Natl Acad Sci U S A 104:11826-11831.

Ross CA, Margolis RL, Reading SA, Pletnikov M, Coyle JT (2006) Neurobiology of schizophrenia. Neuron 52:139-153.

Rubin BD, Katz LC (1999) Optical imaging of odorant representations in the mammalian olfactory bulb. Neuron 23:499-511.

Scheff SW, Price DA (2006) Alzheimer's disease-related alterations in synaptic density: neocortex and hippocampus. J Alzheimers Dis 9:101-115.

Schwaerzel M, Heisenberg M, Zars T (2002) Extinction antagonizes olfactory memory at the subcellular level. Neuron 35:951-960.

Seki Y, Rybak J, Wicher D, Sachse S, Hansson BS (2010) Physiological and morphological characterization of local interneurons in the Drosophila antennal lobe. J Neurophysiol 104:1007-1019.

Selkoe DJ (2002) Alzheimer's disease is a synaptic failure. Science 298:789-791.

Shang Y, Claridge-Chang A, Sjulson L, Pypaert M, Miesenböck G (2007) Excitatory local circuits and their implications for olfactory processing in the fly antennal lobe. Cell 128:601-612.

Shepherd GM (1993) Principles of specificity and redundancy underlying the organization of the olfactory system. Microsc Res Tech 24:106-112.
Sitaraman D, Zars M, Laferriere H, Chen YC, Sable-Smith A, Kitamoto T, Rottinghaus GE, Zars T (2008) Serotonin is necessary for place memory in Drosophila. Proc Natl Acad Sci U S A 105:5579-5584.

Spires TL, Hyman BT (2004) Neuronal structure is altered by amyloid plaques. Rev Neurosci 15:267-278.

Stocker RF, Heimbeck G, Gendre N, de Belle JS (1997) Neuroblast ablation in Drosophila P[GAL4] lines reveals origins of olfactory interneurons. J Neurobiol 32:443-456.

Su CY, Menuz K, Carlson JR (2009) Olfactory perception: receptors, cells, and circuits. Cell 139:45-59.

Tanaka NK, Ito K, Stopfer M (2009) Odor-evoked neural oscillations in Drosophila are mediated by widely branching interneurons. J Neurosci 29:8595-8603.

Uchida N, Takahashi YK, Tanifuji M, Mori K (2000) Odor maps in the mammalian olfactory bulb: domain organization and odorant structural features. Nat Neurosci 3:1035-1043.

Wachowiak M, Cohen LB (2001) Representation of odorants by receptor neuron input to the mouse olfactory bulb. Neuron 32:723-735.

Wagh DA, Rasse TM, Asan E, Hofbauer A, Schwenkert I, Dürrbeck H, Buchner S, Dabauvalle MC, Schmidt M, Qin G, Wichmann C, Kittel R, Sigrist SJ, Buchner E (2006) Bruchpilot, a protein with homology to ELKS/ CAST, is required for structural integrity and function of synaptic active zones in Drosophila. Neuron 49:833-844.

Wang JW, Wong AM, Flores J, Vosshall LB, Axel R (2003) Two-photon calcium imaging reveals an odor-evoked map of activity in the fly brain. Cell 112:271-282.

Watts RJ, Schuldiner O, Perrino J, Larsen C, Luo L (2004) Glia engulf degenerating axons during developmental axon pruning. Curr Biol 14:678-684.

Wilson RI, Laurent G (2005) Role of GABAergic inhibition in shaping odorevoked spatiotemporal patterns in the Drosophila antennal lobe. J Neurosci 25:9069-9079.

Wilson RI, Turner GC, Laurent G (2004) Transformation of olfactory representations in the Drosophila antennal lobe. Science 303:366-370.

Winther AM, Siviter RJ, Isaac RE, Predel R, Nässel DR (2003) Neuronal expression of tachykinin-related peptides and gene transcript during postembryonic development of Drosophila. J Comp Neurol 464:180-196.

Winther AM, Acebes A, Ferrús A (2006) Tachykinin-related peptides modulate odor perception and locomotor activity in Drosophila. Mol Cell Neurosci 31:399-406.

Wong AM, Wang JW, Axel R (2002) Spatial representation of the glomerular map in the Drosophila protocerebrum. Cell 109:229-241.

Yaksi E, Wilson RI (2010) Electrical coupling between olfactory glomeruli. Neuron 67:1034-1047.

Yokoi M, Mori K, Nakanishi S (1995) Refinement of odor molecule tuning by dendrodendritic synaptic inhibition in the olfactory bulb. Proc Natl Acad Sci U S A 92:3371-3375.

Yu D, Ponomarev A, Davis RL (2004) Altered representation of the spatial code for odors after olfactory classical conditioning; memory trace formation by synaptic recruitment. Neuron 42:437-449. 